\title{
Skin Grafting in Xenopus laevis: A Technique for Assessing Development and Immunological Disparity
}

\author{
Yumi Izutsu ${ }^{1}$ \\ Department of Biology, Faculty of Science, Niigata University, Niigata 950-2181, Japan
}

Skin grafting in the amphibian Xenopus laevis has been used to detect not only allogeneic antigens that differ by minor $\mathrm{H}$ antigens or by one MHC haplotype, but also to detect ontogeny-specific antigens (including both emerging adult- and disappearing larval-specific) during metamorphosis. To understand the mechanisms underlying allogeneic tolerance or immune responses against larval- and/or adult-specific antigens, a complete MHC homozygous, inbred strain is the most appropriate experimental model. The inbred J strain established in Japan is used here. Owing to complete histocompatibility, the inbred J strain shows no grafted skin rejection among the same strain of adult frogs, and its genuine homozygosity was reconfirmed by genomic sequence analysis in 2016. Therefore, the J strain enables immunologists and embryologists to understand evolutionary processes as well as immunological events and tissue remodeling mechanisms present during development. Furthermore, an F1 hybrid between the J strain and a GFP-labeled transgenic line is available from our laboratory and can be used as a model for long-term cell tracking. This protocol explains the methodology for skin grafting in X. laevis to determine immunological discrepancies between the host and donor. It is also possible to trace cell and tissue fates in the hosts during early embryogenesis and during complete development from larvae to adults, which is extremely difficult to perform using other species.

It is essential that you consult the appropriate Material Safety Data Sheets and your institution's Environmental Health and Safety Office for proper handling of equipment and hazardous materials used in this protocol.

RECIPES: Please see the end of this protocol for recipes indicated by $<R>$. Additional recipes can be found online at http://cshprotocols.cshlp.org/site/recipes.

\section{Reagents}

MS222 (3-aminobenzoic acid ethyl ester) (Sigma-Aldrich A5040)

A $1 \%$ stock solution of MS222 is stable for several months when stored in a dark glass bottle at $4^{\circ} \mathrm{C}$. For anesthetization of adult frogs, prepare $0.05 \%$ MS222 solution in $1 \times$ Steinberg's solution containing $50 \mu \mathrm{g} / \mathrm{mL}$ gentamicin.

OCT compound (Sakura 4583)

Paraformaldehyde (4\% in phosphate-buffered saline)

\footnotetext{
${ }^{1}$ Correspondence: izutsu@gs.niigata-u.ac.jp

From the Xenopus collection, edited by Hazel L. Sive.

(C) 2019 Cold Spring Harbor Laboratory Press

Cite this protocol as Cold Spring Harb Protoc; doi:10.1101/pdb.prot099788
} 
Steinberg's solution $(1 \times)<\mathrm{R}>$

To prepare a working Steinberg's solution containing $50 \mu \mathrm{g} / \mathrm{mL}$ gentamicin, add 1/1000 volume of gentamicin stock solution (50 mg/mL [Sigma-Aldrich G1397]) plus an equal volume of $0.2 \mathrm{~N} \mathrm{NaOH}$ to $1 \times$ Steinberg's solution. Check that the $\mathrm{pH}$ is neutral and adjust as needed. Store at $4^{\circ} \mathrm{C}$.

Xenopus laevis (inbred J strain and/or gfp-Tg hybrid line) in rearing containers.

The X. laevis inbred J strain was established by Tochinai and Katagiri (1975). In 1948, four pairs of founder frogs were inbred by gonadotropic hormone-induced mating. In 1973, it was found that these inbred frogs showed no short-term skin graft rejection among siblings of the same strain, indicating immunologically high homogeneity. After repeated single-pair mating for over 21 generations, this inbred line showed no long-term skin rejection (Izutsu and Yoshizato 1993). The detailed history of the J strain is described by Session et al. (2016). The J strain is available from a domestic animal vendor in Japan, Watanabe Zoushoku (http://www5d.biglobe.ne.jp/ 〜zoushoku/top.htm).

The gfp transgenic line (gfp-Tg) was generated in our laboratory by nuclear transplantation using wild-type $\mathrm{X}$. laevis carrying the gfp gene under the control of the Xenopus heat shock promoter (hsp70) (Mukaigasa et al. 2009). The partially inbred F4 gfp-Tg line was obtained by hormone-induced mating; the strongest GFPexpressing individuals were selected from among the siblings of the F3 gfp-Tg line (Otsuka-Yamaguchi et al. 2017). The F1 gfp-Tg hybrid J line can be used as a host for skin grafting experiments, as the I strain donor cells grafted to the hybrid recipients can be easily distinguished after induction of GFP expression by heat shock.

Equipment

Cotton sticks (Johnson and Johnson) (optional; see Step 8)

Digital camera (AxioCam HRc; ZEISS)

Filters $(0.2-\mu \mathrm{m}$ pore size) (Millipore GSWP04700)

Fine forceps (110-mm total length) (Natsume Seisakusho [Tokyo] MA-55) (sterilized with 70\% ethanol)

Glass beakers (200-mL) (Iwaki Glass; Asahi Techno Glass, Tokyo)

Kimwipes

Liquid nitrogen

Microscissors (105-mm total length, 7-mm blade length) (Natsume Seisakusho MB-50-7) (sterilized with $70 \%$ ethanol)

Microscope (Leica M60)

Petri dishes (10-cm) (Corning 353803)

Plastic scale $\left(3 \times 3 \mathrm{~mm}^{2}\right)$

Graph paper can be copied to an overhead projector sheet and cut with scissors to create a plastic scale.

Suture needle with thread (6-0 blue nylon polyamide) (Nescosuture ET0806NA45-KF2)

Water aspirator pump (Sigma-Aldrich)

All treatments should be done aseptically to avoid infection.

1. One to two days before the operation, transfer the frogs to a rearing container of filter-sterilized $(0.22 \mu \mathrm{m})$ or autoclaved tap water. Keep them without feeding until the day of the operation.

2. On the day of the operation, gently wash the frogs in a glass beaker 10 times using sterilized water.

3. Anesthetize the animals by immersing them in a glass beaker containing $0.05 \%$ MS222 solution. The duration of anesthesia should not exceed $30 \mathrm{~min}$.

See Troubleshooting.

4. Place a donor animal on its back on a sheet of Kimwipe of suitable size in a 10-cm Petri dish. Pour ice-cold $1 \times$ Steinberg's solution with gentamicin over the animal until the top of the body is almost immersed in solution.

The Kimwipe sheet prevents the slipping of the donor animal during the operation. 
Sufficient solution is necessary because the skin can easily dry and curl up without contact with water once excised from the donor. Once the skin is curled up, it is difficult to use for grafting.

5. Place a plastic scale $\left(3 \times 3 \mathrm{~mm}^{2}\right)$ on the ventral skin of the donor for reference (Fig. 1A). Excise a 1.5- to $3.0-\mathrm{mm}^{2}$ area of skin from the white-colored donor belly (distinguishable from the dark brown-colored back skin of the host) using fine forceps and microscissors. Leave the piece of skin on the ventral side of the donor until it is transplanted (Fig. 1B).

6. Transfer a host animal to the same dish. Trim the same-size area from the dorsal skin of the host to fit the donor skin graft (Fig. 1C, cyan-dotted area). Remove the trimmed skin from the host immediately and arrange the donor and host adjacent to each other.

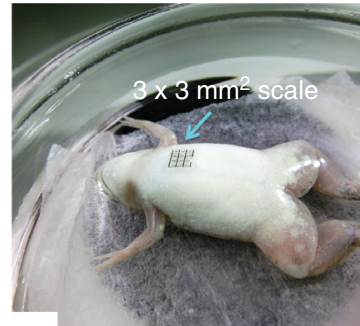

A
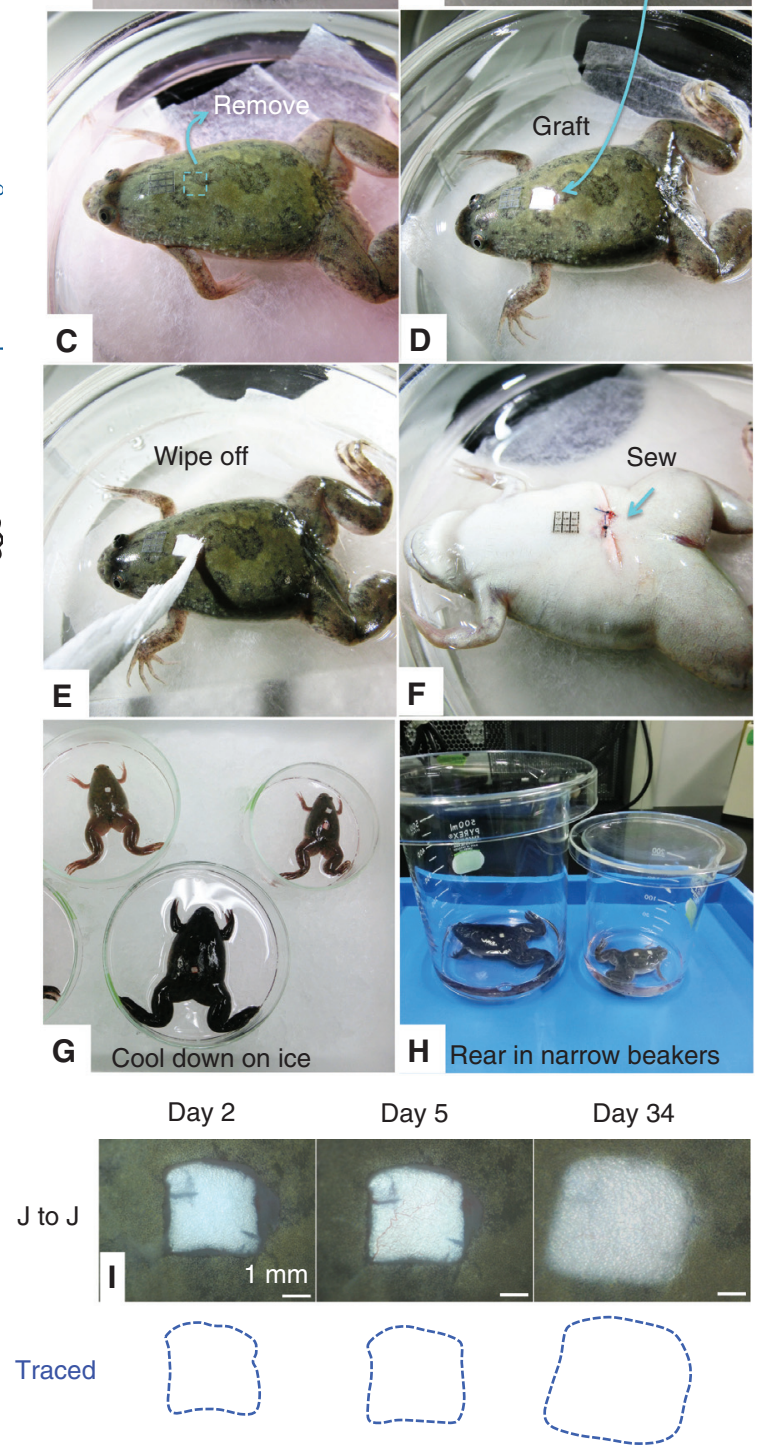

FIGURE 1. Schematic representation of the skin grafting procedure. (A) Anesthetize the J strain donor. Place a plastic scale $\left(3 \times 3 \mathrm{~mm}^{2}\right)$ on the ventral skin. $(B)$ Cut grafts corresponding to the scale. Leave the grafts on the ventral skin. (C) Remove an area of dorsal skin (cyan-dotted area) from the J strain host to create a hole. $(D)$ After making a hole, immediately slide the graft underwater into the hole. Otherwise, the graft will dry and curl up, making it difficult to graft. $(E)$ Carefully wipe the grafted skin to keep the graft dry just after the operation. This treatment raises the success rate dramatically. $(F)$ Use a sutures needle to sew the ventral skin of the donor. (Note that this individual is different from the frog shown in $A$ and $B$.) $(G)$ Keep the hosts on ice to prevent movement. Otherwise, the grafts may become displaced easily. $(H)$ Rear the hosts in narrow glass beakers for 2-3 $d$ to prevent jumping or overturning. (I) After transplantation, once the grafted skin from the white-colored donor belly is accepted by the host frog, it grows as part of the host skin (see Day 34). Blood vessels are observable on Day 5. The grafted skin area is indicated by the traced area (blue-dotted lines) in the images. Tracing is used for image analysis. Note that since the grafted donor skin tissue is completely accepted as self-tissue by the host, the graft continues to develop with the growth of the young host and the boundary between the skin of the white donor and that of the brown host appears blurred and blended. 
7. Add ice-cold $1 \times$ Steinberg's solution with gentamicin to the dish until the tops of both animals are immersed. Transfer the skin graft by gently slipping the donor skin underwater into the graft bed (the hole on the back of the host) using forceps (Fig. 1D).

Care must be taken to not expose the skin graft to air during the operation.

8. After grafting, immediately remove the Steinberg's solution using a water aspirator pump. Wipe off the solution around the graft $>5$ times, particularly at the junction between the graft and the host skin, using a Kimwipe or a cotton stick (Fig. 1E).

This quick wipe-off helps the donor skin adhere firmly to the host skin.

9. If necessary, close the back slit of the donor with sutures (Fig. 1F).

10. Post-operation, move the host frog to a cold $200-\mathrm{mL}$ glass dish or beaker containing a small amount of ice-cold $1 \times$ Steinberg's solution with gentamicin to prevent drying of the animal. Place the dish on ice for $1 \mathrm{~h}$ (Fig. 1G).

The temperature and time required for cooling can be changed depending on the size or stage of the animal.

11. Transfer the dish and incubate for $2-4 \mathrm{~h}$ in the dark at $4^{\circ} \mathrm{C}$.

This prevents the animal from moving and jumping and prevents the grafted skin from flaking off.

See Troubleshooting.

12. Transfer the dish and incubate overnight in the dark at $16^{\circ} \mathrm{C}$.

See Troubleshooting.

13. Rear the host animal in a narrow glass beaker for $2-3 \mathrm{~d}$ at normal temperature $\left(23^{\circ} \mathrm{C}-24^{\circ} \mathrm{C}\right)$

(Fig. $1 \mathrm{H}$ ) with a small amount of $1 \times$ Steinberg's solution with gentamicin.

The narrow glass beaker prevents inversion or jumping.

The Steinberg's solution, which prevents the animal from completely drying up, should be replaced often to prevent infection.

14. Observe the grafted skin using a microscope (Fig. 1I). Confirm that the graft is successfully attached and that its original size (e.g., $3 \times 3 \mathrm{~mm}^{2}$ ) is maintained.

With successful attachment to the host, thin blood vessels extending into the graft should be visible around 2-3 d after grafting (Fig. 11, Day 5).

15. Maintain host frogs at normal temperature $\left(23^{\circ} \mathrm{C}-24^{\circ} \mathrm{C}\right)$ in rearing containers with a suitable volume of dechlorinated tap water, at a maximum density of five frogs per $2 \mathrm{~L}$. Feed frogs and change the rearing water every day.

16. Photograph the host animal grafts every $2-3 \mathrm{~d}$ using a digital camera. Trace the areas of the skin grafts (Fig. 1I, traced).

The traced areas can be measured and compared to their original sizes at Day 1 using the public domain image processing and analysis program "Imagel" (https://imagej.nih.gov/ij/).

17. To determine the cell and tissue fates of the grafted cells, prepare the tissues for immunohistochemistry.

i. Dissect the grafts, including some of the host skin, using fine forceps and microscissors.

ii. Place the skin samples on $0.22-\mu \mathrm{m}$ filter membranes (e.g., $3 \times 3 \mathrm{~mm}^{2}$ ) to keep the skin flat.

iii. Embed the tissues in OCT and then dip in liquid nitrogen.

iv. Fix the tissue sections with $4 \%$ paraformaldehyde for $10 \mathrm{~min}$.

Tissue samples can be prepared any time after proper graft attachment (Step 14), up to 6 mo after grafting. To look for features of skin graft rejection, sections should be prepared when blood vessel numbers increase or inflammation is observed. Migration of effector T cells and degeneration of tissue may be visible.

Sometimes GFP fluorescence, derived from the gfp-Tg line, quenches during immunostaining even after fixation. Therefore, GFP visualization must be performed before immunostaining. Two different colors, e.g., Alexa488 (green)- and Cy3 (red)-conjugated antibodies, may be used. GFP fluorescence can be detected using anti-GFP antibodies. 
Problem (Steps 3, 11, and 12): Animals vomit during the course of grafting.

Solution: If the animals are fully fed, they sometimes vomit during and just after the operation. At least $1 \mathrm{~d}$ before the operation, the animals should be fasted.

\section{DISCUSSION}

Because genomic information is now available for the X. laevis J strain (Session et al. 2016), it is possible to produce genome-edited J strain frogs. Cell and tissue transplantation can be used in animals with the J strain genetic background as an innovative strategy to address unresolved questions about mechanisms of development. These experiments also enable both genetic labeling for cell tracking and ectopic gene induction in the grafted cells. Although the J strain has been inbred for decades, it does not possess any problematic characteristics that might affect experimental planning or development and growth when compared to wild-type frogs. While the body size of the J strain is smaller than that of the wild-type frog at the same age, and while J strain frogs produce a smaller number of eggs, a sexually mature J strain female produces approximately 1000-2000 eggs. During hormone-induced mating, $80 \%-90 \%$ of shed eggs are usually fertilized; therefore, it is easy to produce more than 1000 metamorphosed frogs from one mating. Since the J strain has perfect histocompatibility, it can be used for experiments without the immunological barrier, which is not possible in many other cold-blooded animals.

Steinberg's Solution (1x)

$3.4 \mathrm{~g} / \mathrm{L} \mathrm{NaCl}$

$0.05 \mathrm{~g} / \mathrm{L} \mathrm{KCl}$

$0.08 \mathrm{~g} / \mathrm{L} \mathrm{Ca}\left(\mathrm{NO}_{3}\right)_{2} \cdot 4 \mathrm{H}_{2} \mathrm{O}$

$0.1025 \mathrm{~g} / \mathrm{L} \mathrm{MgSO} 4$

$0.56 \mathrm{~g} / \mathrm{L}$ Tris- $\mathrm{HCl}(\mathrm{pH} 7.4)$

$10 \mathrm{mg} / \mathrm{L}$ phenol red

For convenience, prepare a $10 \times$ stock solution. Store the $10 \times$ stock solution for no longer than a few months at room temperature.

\section{ACKNOWLEDGMENTS}

I thank Dr. Shin Tochinai for the critical reading. I also thank Haruka Kobayashi and Akira Nakamura for technical assistance. Work in my laboratory is supported by Grant-in-Aid from Nagase Science Technology Foundation (to Y.I.) and by Grant-in-Aid for Scientific Research (C) from the Ministry of Education, Culture, Sports, Science, and Technology (15K06992 to Y.I.).

\section{REFERENCES}

Izutsu Y, Yoshizato K. 1993. Metamorphosis-dependent recognition of larval skin as non-self by inbred adult frog (Xenopus laevis). J Exp Zool 266: 163-167.

Mukaigasa K, Hanasaki A, Maéno M, Fujii H, Hayashida S, Itoh M, Kobayashi M, Tochinai S, Hatta M, Iwabuchi K, et al. 2009. The keratin-related Ouroboros proteins function as immune antigens mediating tail regression in Xenopus metamorphosis. Proc Natl Acad Sci 106: 18309-18314.

Otsuka-Yamaguchi R, Aiko Kawasumi-Kita A, Kudo N, Izutsu Y, Tamura K, Yokoyama H. 2017. Cells from subcutaneous tissues contribute to scarless skin regeneration in Xenopus laevis froglets. Dev Dyn 248: 586-597.

Session AM, Uno Y, Kwon T, Chapman JA, Toyoda A, Takahashi S, Fukui A, Hikosaka A, Suzuki A, Kondo M, et al. 2016. Genome evolution in the allotetraploid frog Xenopus laevis. Nature 538: 336-343.

Tochinai S, Katagiri C. 1975. Complete abrogation of immune response to skin allografts and rabbit erythrocytes in the early thymectomized Xenopus. Dev Growth Differ 17: 383-394. 


\section{Skin Grafting in Xenopus laevis: A Technique for Assessing Development and Immunological Disparity}

Yumi Izutsu

Cold Spring Harb Protoc; doi: 10.1101/pdb.prot099788 originally published online January 3, 2019

\begin{tabular}{rc}
$\begin{array}{r}\text { Email Alerting } \\
\text { Service }\end{array}$ & Receive free email alerts when new articles cite this article - click here. \\
\hline $\begin{array}{c}\text { Subject } \\
\text { Categories }\end{array}$ & $\begin{array}{c}\text { Browse articles on similar topics from Cold Spring Harbor Protocols. } \\
\text { Developmental Biology (728 articles) } \\
\text { Immunohistochemistry (92 articles) } \\
\text { Immunology, general (128 articles) } \\
\text { Xenopus (210 articles) } \\
\text { Xenopus Transgenics (32 articles) }\end{array}$ \\
\hline
\end{tabular}

